\title{
Two Physical Constraints upon the Motions of Celestial Bodies
}

\author{
Xiaobai Ai \\ Shanghai Institute of Applied Physics, The Chinese Academy of Sciences, Shanghai, China \\ Email: hsiaobaiai@outlook.com, mraixiaobai@163.com
}

How to cite this paper: Ai, X.B. (2019) Two Physical Constraints upon the Motions of Celestial Bodies. Journal of Modern Physics, 10, 344-361.

https://doi.org/10.4236/jmp.2019.103023

Received: January 24, 2019

Accepted: March 11, 2019

Published: March 14, 2019

Copyright $\odot 2019$ by author(s) and Scientific Research Publishing Inc. This work is licensed under the Creative Commons Attribution International License (CC BY 4.0).

http://creativecommons.org/licenses/by/4.0/

\section{c) (i) Open Access}

\begin{abstract}
There exist two physical constraints upon the motions of celestial systems. Constraint 1 reveals during collapse or explosion motion of celestial bodies that there would be an unattainability upper limit for their compact intensity (total mass $M$ /scale size $R$ ), which arises from the Lorentz invariance of the time-like metric in local four-dimensional continuum in Einstein's theory of special relativity. Constraint 2 points that the average mass density of nucleon would be an unsurpassed upper limit for bulk normal matter in nature, which arises from Heisenberg's uncertainty principle. A very important effect is that the combination of these two physical constraints would prevent the formation of black holes.
\end{abstract}

\section{Keywords}

General Theory of Relativity, Gravitation Collapsing, Schwarzschild Metric, Schwarzschild Singularity, Centre Singularity, Black Hole, Compact Intensity, Heisenberg's Uncertainty Principle

\section{Introduction}

Hawking (1942-2018) in his last few years continued to issue negative voices on the black hole theory [1] [2] that he had participated in as a leading scholar. However, the lack of convincing physical arguments is a prominent weakness in his last works, especially in the "smooth inflation exiting" article written by Hawking, S. and Hertog, T. [2]. Many physicists believe that their new idea was not practical: "their research results being in an empty mathematical framework", "ignoring many details of the development of the universe", "only being speculations without any practical basis". Other scholars who did deny the black hole theory have similar deficiencies, for instance, see [3] [4].

It is well known that since 1916 Schwarzschild firstly solved a solution of 
Einstein's gravitational field equation for outside a static and spherically symmetric vacuum field with central mass $M$ i.e. the Schwarzschild metric:

$$
\mathrm{d} s^{2}=c^{2}\left(1-\frac{2 G M}{R c^{2}}\right) \mathrm{d} t^{2}-\left(1-\frac{2 G M}{R c^{2}}\right)^{-1} \mathrm{~d} R^{2}-R^{2}\left(\sin ^{2} \theta \mathrm{d} \phi^{2}+\mathrm{d} \theta^{2}\right),
$$

where $r, \theta, \phi$ are the usual polar coordinates, $G$ being the constant of gravitation, $R$ is the scale of radius, $c$ is the speed of light in vacuum. If there are no physical constraints, there would be two singularities in Equation (1): the singularity of Schwarzschild $\left(R_{S}=c^{2} / 2 G\right)$ and the singularity of centre $(R=0)$. In the 1920s, some famous physicists, including Einstein and Eddington, did not believe the outcome from Equation (1) and a used famous opinion was that "there should be certain laws in nature to prevent the stars from evolving into such absurd outcomes". But scholars had been looking for the laws over a decade, and didn't still know what they were. Therefore, during an unstoppable gravitational collapse, the formation of singularities would be inevitable physical state.

By the 1930s, there seemed to be a consensus in astronomical community that the singularity of Schwarzschild could be eliminated by re-selecting coordinates. In 1933, Lemaitre, G. used a moving metric, the infinite red-shift horizon was eliminated, but the white hole would be created [5]. In 1935, Einstein and Rosen showed that they applied the Einstein-Rosen Bridge (or Schwarzschild wormhole) to replace the singularity of Schwarzschild [6]. During 1960, Kruskal applied the dynamic metric instead of Schwarzschild coordinate, then the space-time would be divided into four regions, and the white hole would appear [7]. On the surface of the collapsing body, they used coordinate transformations to eliminate Schwarzschild singularity. In fact, they just used new puzzles instead of old ones. Almost at the same time, Finkelstein wanted to use the Eddington-Finkelstein coordinates to eliminate the singularity of Schwarzschild, but he gave birth to the white hole monster [8]. Mei, X.C. pointed out that "black holes with singularities" "caused by the mathematical description of curved space-time", but he could not give the physical principles to study gravitational collapse only in the flat space-time [9] [10] [11].

Recently the most sensational event for physical world is that physicists in United States and Europe have announced that: from February 11, 2016 to August, 2017, they have observed GW150914, GW151226, GW170104 and GW170817 four gravitational wave (GW) events. In 2017, the Nobel Prize in Physics was awarded to Weiss, R., Thorne, K. and Barish, B. for their role in the direct detection of GW. It is a firm fact that GW has been detected. Taking GW150914 [12] [13] as an example which has been modeled with the techniques of numerical relativity [14] [15] [16], the GW is strictly credible to be measured, but the resulting analysis confirmation is not yet convincing and still in doubt. Question is mainly from two points: a) The numerical simulation method usually uses preconceived premises to construct the model, then the "computer experiment" is used to adjust repeatedly until the parameters suitable for or matching the observed data would be found. The existence of black holes in nature is the premise 
of numerical simulation, if there are no black holes in nature world, what shall we do? It is a pity, the published results of GW150914 lacked key data to confirm black holes, i.e. lacked the scales $R_{1}$ of $M_{1}\left(29 M_{\odot}\right)$ and $R_{2}$ of $M_{2} \quad\left(36 M_{\odot}\right)$ from the parameters provided in the analysis paper [12] [13]. The scale of $62 M_{\odot}$ might not be obtained until the final merging stage. In short, it is very important to get $M_{1} / R_{1}$ and $M_{2} / R_{2}$ firstly. b) It is generally believed that the energy of gravitational wave comes from the sum of the conversion of various kinetic energy and the release of gravitational binding energy from collapsing celestial bodies which would have a higher conversion efficiency [17]. Gursky, H. toke a single proton as an example and pointed out that in [18]: "We can get a clue by calculating the potential energy of a single proton on the surface of stars of decreasing size but of $1 M_{\odot}$, thus of increasing density, as given by

$$
\phi=\frac{G M_{\Theta} m_{p}}{R}=G M_{\Theta}^{2 / 3} \rho^{1 / 3} m_{p} .
$$

Hence, even if a single proton landed on the surface of stars might release all its gravitational binding energy, which would be still less than half of its rest energy. So do other materials.

Consequently, in [12] and [13], a small part of the energy of $3.2 M_{\odot} c^{2}$ radiates as a gravitational wave, but where is the most part of the matter of $3.2 M_{\odot}$ ? Or where do the most part of the matter of $3.2 M_{\odot}$ go?

Because gamma-ray bursts were not monitored synchronously in the final merger stage and subsequently no gamma-ray burst were detected [12]. Is there a new pattern of material disappearance to be recognized? Or through some unknown mechanism, $3.2 M_{\odot}$ of matter has been converted into the energy of a short gamma-ray burst and the afterglow which have been missed? Obviously some alternative conclusion may be considered also. A temporary and more reasonable explanation for the gravitational wave event GW150914 should be that a pair of merged objects with $29 M_{\odot}$ and $36 M_{\odot}$ merging into a single collapsed object with $62 M_{\odot}$, and a small part of the energy of $3.2 \mathrm{M}_{\odot} c^{2}$ has been radiated as a gravitational wave in the following "circular" motions. The above arguments are also applicable to the events of GW 151226, GW 170104 and GW 170817.

Recently, the STAR Collaboration at Brookhaven National Laboratory's Relativistic Heavy Ion Collider (RIHC) measured the polarization of the decay products of $\Lambda$ hyperons which are emitted from the quark-gluon plasma (QGP) produced by ultra-relativistic collisions between heavy atomic nuclei traveling close to the speed of light and has determined the rotational speed of QGP were about $(9 \pm 1) \times 10^{21}$ times/sec [19]. Scientists at the Thomas Jefferson National Accelerator Institute in the United States found, firstly, that the pressure in the center of the proton is around $10^{35} P_{a}$ (i.e. $10^{36} \mathrm{erg} / \mathrm{cm}^{3}$ ) [20]. Although with larger statistical uncertainties, the high rotation rate of bulk QGP material and high pressure states resemble white dwarfs and neutron stars, hence [19] and [20] not only provide experimental insights into the physics of the strong nuclear 
force, but also forces us to ponder: because of $\omega R<c$, where $\omega$ being is the rotation rate of QGP in [19], as long as the natural normal matter, such as elementary particles, has a geometric scale R, [19] and [20] would imply that, for the high-density normal material in nature, there would exist some upper limits of physical property such as the material density, the energy density, high pressure and etc. which would not be reached or be unsurpassed. Anyhow, [19] and [20] prompt the author to re-examine the theory of relativity and quantum mechanics and to find that the keys to unlock the mysteries of black hole would hide in our neglected life experience and the careless research style.

In Section 2, the present author argues that at least two criteria might be used to judge whether an observed celestial body being as a "black hole candidate". In Section 3, the careless style of study and some neglected experiences in previous studies are pointed. In Section 4, the first restriction on the motions of celescial systems is derived from the Lorentz invariance of the time-like metric in local four-dimensional continuum in Einstein's theory of special relativity (SR) which prevents the gravitational collapse of compact object reached general relativistic instability stage from Schwarzschild radius. In Section 5, the second restriction on the motions of celescial systems is derived from Heisenberg's uncertainty principle which reveals that the mean density of normal matter in nature has an unsurpassed upper limit. In Section 6, the new physics included in Constraint 1 and Constraint 2 will be discussed. In Section 7, some remarks are given.

\section{Available Criterion}

If the gravitational collapse to a black hole is unavoidable during the late evolution of a massive celestial body, then observers standing outside and far away from Schwarzschild radius will never be able to observe it, the reason was pointed out by Weinberg G.: "The collapse to the Schwarzschild radius therefore appears to an outside observer to take an infinite time" [21]. As we all know, the collapsing celestial body still has a strong gravitational field and during the collapsing process there will be a variety of external substances that will continue to be pulled into. Hence the community of astronomy and physics are still concerned about whether indirect evidence of the existence of black holes can be obtained from astronomical observations. Therefore, many indirect methods to identify black holes were proposed: such as Hawking's black hole radiation; the gravitational waves generated by the non-spherical symmetric component of gravitational field changing sharply with time or a merger of two celestial bodies; X-ray or X-ray bursts from accretion disks around black holes; radiations or emissions of matter from the accretion disk falling into a black hole, and so on. Unfortunately, these phenomena of expectation are only the criteria for pre-selection, but the credible evidence has not yet been found, because the similar phenomena may also occur in the movement of other celestial bodies. However, if one wants to deny many candidates for black holes, there exists available compact intensity $(M / R)$ criterion. 
For an unknown compact celestial body, its total mass $M$ and its scale of radius $R$ (or diameter $2 R$ ) usually are measured firstly. According to Equation (1), if celestial bodies can reach or break through Schwarzschild radius in gravitational collapse, the compact intensity $(M / R)$ should be

$$
\frac{M}{R}>\frac{c^{2}}{2 G}\left(\approx 6.75 \times 10^{27} \mathrm{~g} / \mathrm{cm}\right) \text {. }
$$

Otherwise, some material or most material of the candidate would be still outside of "Schwarzschild radius" of an expected black hole. Hence, the magnitude of $M / R$ should be an important criterion.

Example 1: The giant elliptical galaxy Messier 87 (M87) at the center of the Virgo cluster of galaxies is a strong radio source, an active galaxy and a very strong source of gamma rays. The Hubble Faint Object Spectrograph (FOS) was used to measure the rotation velocity of the ionized gas disk at the center of M87, the FOS data indicated a central mass of 2.4 billion solar masses, with $30 \%$ uncertainty [22]. In the past, M87 was thought as a 50 billion solar masses center span about 300 light-years across. Recently, using the High Energy Stereoscopic System Cherenkov telescopes, scientists measured the variations of the gamma ray flux coming from M87 and found that the flux changed over a matter of days, which might suggest the diameter of M87 being only round 0.1 ly [23]. Even so, the evaluating values of compact intensity is only $M / R \approx 1.0 \times 10^{26} \mathrm{~g} / \mathrm{cm}$, in fact, which does not support M87 being a reliable candidate of the black hole.

Example 2, let us Sagittarius $A^{\star}$ at the centre of our Milky Way. [24] gave that Sagittarius $\mathrm{A}^{\star}$ weights 4.3 million times of the Suns, [25] gave that an intrinsic size of the radio-emitting region of the galactic center compact non-thermal radio source of only $1 \mathrm{AU}$ in diameter at $3.5 \mathrm{~mm}$. For $1 \mathrm{AU} \approx 1.496 \times 10^{13} \mathrm{~cm}$, the evaluating results are: $M / R \approx 1.14 \times 10^{27} \mathrm{~g} / \mathrm{cm}$, the mean density $\rho \approx 4.8 \mathrm{~g} / \mathrm{cm}^{3}$. At least, the present measured result of $M / R$ does not support Sagittarius $A^{*}$ being a reliable candidate of a super-massive black hole. May be, a consolation argument says: the radio-emitting region being outside the center of "black hole", the gravitational collapse in Sagittarius $A^{*}$ still continues, its scale $\mathrm{R}$ would be further reduced and more accurate measurements should be awaited in the future. However, the negative opinions will be supported by another two astronomical observations.

\section{a) Red-shift}

If there is a black hole, the gravitational potential energy near the event horizon varies greatly. It is generally believed that the linear spectrum of iron may be detected in the region closest to the black hole. More scholars estimate that $\mathrm{X}$-ray or gamma radiation should be the dominant radiation near the event horizon. In fact, if radiation would be measured, regardless of the form of radiation, the gravitational red shift value must be considerable. Therefore, the value of gravitational red-shift should be another important criterion.

The compact intensity $M / R$ might be expressed in terms of gravitational red-shift as 


$$
\frac{M}{R}=\frac{c^{2}}{2 G}\left[1-\frac{1}{(1+z)^{2}}\right],
$$

where $z$ is red-shift which may be evaluate by

$$
z=\frac{\lambda-\lambda_{0}}{\lambda_{0}}=\frac{\lambda}{\lambda_{0}}-1,
$$

where $\lambda_{0}$ is the characteristic wavelength of the emitter and $\lambda$ the observed apparent wavelength respectively. If there are no spectral lines of distinguishable wavelengths, the red shift should be calculated by energy also:

$$
1+z=\frac{\lambda}{\lambda_{0}}=\frac{v_{0}}{v} \rightarrow \frac{\hbar v_{0}}{\hbar v}=\frac{E_{0}}{E},
$$

where $E_{0}$ is the energy of the emitting radiation and $E$ the energy of observed radiation respectively. One may see that as long as the measured red-shift $z$ is a limit value, which would only indicate that the scale size of the collapsing bodies is always greater than "Schwarzschild radius".

As an example, let us consider a quasar with a large red-shift value. For $z \geq 0.25$, the red-shift-apparent visual magnitude diagram in [26] displays dispersion, which seems to imply that there is no correlation between apparent visual magnitude and red shift, which is very different from a better linear distance-red-shift relation for $z$ in the range from 0.003 to 0.2 [27]. As a matter of fact, it only implies that: i) the simple linear Hubble's law no longer gives the correct distant radical scale; ii) the dispersion in red shift-apparent magnitude diagram in [26] means that for distant objects with $z \geq 0.25$ there exists several very different physical red shift mechanisms: cosmological red-shift, gravitational red-shift, due to high speed ejecting, the influence of the deceleration parameter $q_{0}$ in cosmology, the effect of evolution and some new kind principles of physics. Hence, regardless the details of debate about the nature of the red shifts of the distant objects, the gravitational red-shifts would be always involved. As long as the above arguments are accepted, even with red-shift greater than 0.25 , the core of the distant celestial objects cannot be associated with any black hole model. For example, S50014 + 813 with 40 billion solar masses [28] and $z \approx 3.366$ [29] [30], its gravitational red-shift must undoubtedly be less than 3.366. If a misuse of $z \approx 3.366$ as its gravitational red shift to estimate $\frac{M}{R} \approx 6.39 \times 10^{27} \mathrm{~g} / \mathrm{cm}$, still $<6.75 \times 10^{27} \mathrm{~g} / \mathrm{cm}$.

Generally speaking, As long as the red shift data are available, it would just prove that the scale of the collapsed object is still larger than "Schwarzschild radius $R_{S}\left(=c^{2} / 2 G\right)$ ".

\section{b) X-ray or Gamma ray}

Can we determine the physical properties of the sources from the characteristics of the energy spectra of the measured X-rays or gamma rays? May the measured X-rays or gamma rays radiations be used as signs for black hole candidates or other origins? The mechanisms of gamma-ray emission are diverse, including electron-positron annihilation, the inverse Compton Effect, from the 
gamma decay of radioactive nuclei in supernovae, hyper-novae, pulsars, blazars and active galaxies and etc. Of course the energy spectra from compact objects and their surrounding environments are cluttered and difficult to measure, even the highest photon energy reaches the $\mathrm{TeV}$ level, but the energy spectra of gamma rays produced from the gravitational collapse would still regularly searchable. For example, proton fall to a star, the more regular energy spectra around 100 $\mathrm{keV}$ implies that radiations may come from white dwarfs; $100 \mathrm{MeV}$ radiations from neutron stars, $300-400 \mathrm{MeV}$ radiations might produced in more compact object-quark star (or called QGP star) that has been speculated for a long time. In fact, as long as the energy spectra of regular rays can be distinguished from the measured messy spectra, whether it is about $100 \mathrm{MeV}$ or $300-400 \mathrm{MeV}$, which would certainly mean that the scale size of the collapsing celestial object is still greater than Schwarzschild radius.

\section{Deficiencies in Previous Studies}

As we all know, Minkowski delivered his famous "space-time" speech at the Conference of Scientists held in Cologne on September 21, 1908 [31]. In this speech, Minkowski extended Einstein's theory of special relativity (SR) [32] to space-like $\left(\mathrm{d} s^{2}<0\right)$, light-like $\left(\mathrm{d} s^{2}=0\right)$ as well as time-like $\left(\mathrm{d} s^{2}>0\right)$ three statuses for space-time continuum, where $\mathrm{d} s^{2}=c^{2} \mathrm{~d} t^{2}-\mathrm{d} x^{2}-\mathrm{d} y^{2}-\mathrm{d} z^{2}$. Later, Einstein accepted Minkowski's contribution and laid the foundation of general theory of relativity (GR) and he pointed out in his book "The Meaning of Relativity" wrote in 1922, that "As in the special theory of relativity, we have to discriminate between time-like and space-like line elements in the four-dimensional continuum" [33]. I don't know why the predecessors, including Einstein himself, forgot this statement, which contained the power they were looking for to prevent the gravitational collapsing of catastrophe. When applying GR to study the gravitational problems, is there any guarantee of physical laws to implement Einstein's "distinction"? The answer is yes, because human beings have not only thousands of years of practical experience, but have GR already.

\section{Practical Experience:}

1) Since the beginning of human ancestors, we have been pursuing the universality of natural laws and have realized the universality from the practice of thousands of years and astronomical observation in different places on the earth. In the progress of the civilization, we have far more knowledge of astronomy than our ancestors from four channels for astronomical information: electromagnetic radiation, cosmic ray particles, neutrinos (anti-neutrinos) and gravitational waves. In addition to extreme physical states, we have thought that, the production mechanism of those events happened in distant celestial bodies would be contained within the physical laws obtained by various research institutions (local inertial systems) on Earth. In other words, at present, we have applied the knowledge of physics obtained in local inertial systems on Earth to study events occurring on distant celestial bodies. Above historical experience 
should not be forgotten, the summary of worth recalling is roughly as follows:

Particle Physics $\rightarrow$ origin of the universe, dark matter, compact stars, active galactic nuclei, supernova explosions and etc.;

Nuclear Physics $\rightarrow$ elemental origins, star structure and evolution, neutron star cooling, solar and cosmic neutrinos, cosmic rays and etc.;

Atomic, Molecular $\rightarrow$ interstellar molecules, astrochemistry, interstellar microwave sources and etc.;

Plasma and Beam Physics $\rightarrow$ radiation power structures, Supernova remnants, pulsar magnetosphere, star wind, cosmic particle acceleration mechanism and etc.;

Condensed Matter $\rightarrow$ interstellar dust, Nebula, white dwarf structure, neutron star structure and etc.;

...

and so on and so forth.

The most gratifying thing is that bold use has achieved a great success. This point proves that in any space locality or time period in the universe, no matter the event being a slow process or a violent explosion, which would be considered as an event occurring in a flat local inertial system.

2) As we all know, matter is not only the basic substance of physics, but also includes measurable electromagnetic field, gravitational field and vacuum field of various virtual particles which cannot be measured directly. However, since there is no indisputable evidence to hint that those accompaniments such as electromagnetic field, gravitational field and/or background vacuum field do take part into gravitational collapse, consequently, during the catastrophic gravitational collapsing processes, only those wrecks with non-zero rest mass torn apart by tidal action such as "dust" grains, gas-dust complex, material molecular clusters, nuclei, and individual elementary particles are the leading role. So that the universal speed limit in Einstein's time-like SR must be considered. In other words, we must adhere the Lorentz invariance of Minkowski local time-like metric as one of the axioms needed in studying the collapsing or exploding process.

\section{Un-negligible Theoretical Pillar:}

1) Let's think about the progress of physics from the perspective of epistemology.

It took about 300 years to express the universality of knowledge in theory and practice. Because the success of electromagnetic field theory promoted the development of Galileo's principle of relativity (Galileo transformation) into Einstein's principle of relativity (Lorentz transformation), and then to inertia relativistic principle (the principle of equivalence), which led to the birth of GR. In this process, it was found that there were many restrictive functional relationships (relativistic effects) between physical quantities. It was found that algebra and geometry may be used as mathematical tools to express physical laws equivalently. Of course, algebra expressions and the algebraic expressions of geometry concepts have their own characteristics, but just being tools. GR is generally 
regarded as a satisfactory modern theory of gravity with tensor potential. In fact, the greatest contribution of GR to whole (!) physics system is to expound and to prove that any locality in the universe would be a flat inertial system, where any laws of physics found on Earth could be applied. This is the theoretical pillar of using the laws of physics found in earth within the mass and distance scales of the universe.

2) SR is not a special branch of physics like nuclear physics, particle physics and etc., but the restrictive relationship between physical quantities revealed by SR would apply to all branches of physics. Einstein and many physicists modified mechanics, optics, electrodynamics, statistical mechanics and quantum mechanics with the constraints of these discoveries, so that the original branches of physics may be applied to low-speed and high-speed motion conditions, as well as physical states under extreme conditions. The establishment of GR seems to expand the concept of inertial system, but it also reduces the concept of inertial system to locality. However, we may rest assured that SR would be used in any processes of any locality in our observable universe. One may see that the rest of this article will prove that the story of black holes should be rewritten, because the scale size of collapsing objects would be always larger than the old concept-Schwarzschild radius.

\section{Constraint 1}

This section deals with another feature of the axiomatic system. The SR and the GR are axiom systems, which are the systems with formal logic. All the theoretical inferences are included in their premises and are determined uniquely by its premises with consistence, independence as well as completeness. From the arguments mentioned in Sections (2) and (3), one may see that without the limitations of certain physical principles, the use of GR will inevitably lead to the infinite collapse of late celestial bodies in evolution, firstly crossing the Schwarzschild radius and until to the singularity of centre $(R=0)$. However, coupled with the Lorentz invariance of Minkowski's time-like metric, some new physical results different from the current black hole theory should be obtained.

Combine Equation (1) and condition $\mathrm{d} s^{2}>0$, one may have an algebraic equations (7) with a "greater sign (>)":

$$
c^{2}\left(1-\frac{2 G M}{R c^{2}}\right) \mathrm{d} t^{2}>\left(1-\frac{2 G M}{R c^{2}}\right)^{-1} \mathrm{~d} R^{2}-R^{2}\left(\sin ^{2} \theta \mathrm{d} \phi^{2}+\mathrm{d} \theta^{2}\right) .
$$

In order to keep the inequality direction of Equation (7), the following positive condition are necessary:

$$
1-\frac{2 G M}{R c^{2}}>0,
$$

or 


$$
R>\frac{2 G M}{c^{2}}
$$

or

$$
M / R<c^{2} / 2 G\left(\approx 6.75 \times 10^{27} \mathrm{~g} / \mathrm{cm}\right) .
$$

Thus, the first constraint upon the motion of late celestial bodies may be obtained naturally. Obviously, this restriction eliminates the singularity of Schwarzschild:

Constraint 1-During collapse or explosion motion of celestial bodies, a special constant $c^{2} / 2 G$ is an unattainability upper limit of the compact intensity $M / R$, i.e. $M / R<c^{2} / 2 G\left(\approx 6.75 \times 10^{27} \mathrm{~g} / \mathrm{cm}\right)$.

As long as to note that comparing with $M$ and $R$, such as angular momentum $J$, charge $Q$ and cosmic constant $L$ involved in other metrics are negligible physical quantities, so that, the above Constraint 1 would apply not only to Schwarzschild metric, but also to other metrics, such as Kerr metric and etc.

According to Equation (10), the following properties of average density may be obtained:

$$
\rho_{m} M^{2}<\frac{3 c^{6}}{32 \pi G^{3}} \approx 7.3 \times 10^{82}(\mathrm{~g} / \mathrm{cm})^{3},
$$

where $\rho_{m}$ is mass density. If change $\rho_{m}$ to energy density $\rho_{E}, E$ is total energy, then

$$
\rho_{E} E^{2}<\frac{3 c^{12}}{32 \pi G^{3}} \approx 5.3 \times 10^{145}(\mathrm{erg} / \mathrm{cm})^{3} .
$$

One may find that the motion of celestial bodies is governed by Equations (11) and (12). A derivable conclusion beyond expectation is that Constraint 1 also reveals that during the process of an expansion or an explosion of celestial body system, the continuous creation of matter from vacuum (?) is inevitable. It is really "something comes from nothing"! On the contrary, in the process of collapse, with the increase of mass density and energy density it would lead to the decrease of total mass (energy). Except various radiations (including infrared, optical, radio, X-ray and gamma rays), energetic particles or spurted material (e.g. the jets) ejected from the accreted disks and gravitational waves generated by the release of gravitational binding energy etc., a new losing way of mass unfolds, because the disappearance of $3.2 \mathrm{M}_{\odot}$ in [12] and [13] is apparently proceeding rapidly through some unknown mechanisms. That is to say, as long as the Equations (11) and (12) are met, the matter should continuously disappear into the vacuum, "something goes back to nothing", which might also be a reasonable physical channel. Maybe a very short gamma-ray burst would originate here. What actually happens? This should be confirmed by the energy spectra of short gamma-ray bursts.

It should be recalled that Weinberg used to have a similar result $(M G / R)<4 / 9$ [i.e. $M / R<0.89 c^{2} / G$ ] [34]. Although Weinberg's upper limit was slightly different from Constraint 1, but careful reading [34], one may find that in order to 
obtain $(M G / R)<4 / 9$, Weinberg used a non-physical "to have $r_{\infty}^{2}$ negative" condition (i.e. imaginary value of point). As one read the following words: "It is difficult to imagine that a fluid sphere with a large density near the surface than near the center could be stable" [35], one may know that Weinberg still considered the infinite collapse, hence, his mistakes in [34] [35] are obvious.

Some people once believed that the contribution of variables to mathematics was attributed to Descartes, R. In fact, when the sign of inequality entered mathematics, the variables, the motion of variables, the moving direction of variables and the limit of motion were all brought into mathematics. All physical equations expressed by inequalities imply that there must be "unattainable" or "insurmountable" restrictions on a certain physical state. It is noteworthy that conditional Equations (3) and (10) have dynamic characteristics already, and the calculated Schwarzschild radius would be a variable now and only with instantaneous value. However, "Constraint 1" and Equation (11) would still not prevent collapse, but only impose one strong restriction on the collapsing of compact bodies. According to Equation (11), even for late evolving celestial bodies, if the collapsing time is long enough, their volume will collapse to a small enough size, and it seems inevitable that they would still collapse to a state with very high mass density, even onto or pass Schwarzschild singularity. In fact, this is impossible, because the new achievement of high energy physics [19] implies that Heisenberg's uncertainty principle will give one more physical constraint.

\section{Constraint 2}

A quark-gluon plasma (QGP) or quark soup is a state of matter in quantum chromodynamics (QCD) which exists at extremely high temperature and/or density. This state is thought to consist of asymptotically free strong-interacting quarks and gluons, which are ordinarily confined by color confinement inside atomic nuclei or other hadrons. Although they are all complex QCD systems [36], the order of magnitude of their bulk properties may still be estimated by basic natural constants.

According to the theoretical principle, the density of micro QGP in [19] might be estimated from its rotational speed data. Because of the universal speed limit given by time-like SR, the upper limit on the rotational speed of micro QGP would mean that there should be an upper limit on the mass density and energy density of micro QGP. Obviously, the formation mechanism of micro QGP might be different from that of white dwarfs, neutron stars and the presumed quark stars (QGP stars). The former is formed by strong-interaction, the latter by gravitation. Therefore, for micro QGP, there might be a "strong interaction gravitational constant" $G_{S}$ similar to the gravitational constant G. The author will present a detailed discussion of this topic at a later date.

Let us turn to see [20]. The pressure in the center of the proton was measured around $P=10^{35} P_{a}$ (i.e. $10^{36} \mathrm{erg} / \mathrm{cm}^{3}$ ) [20], its mass density $\rho_{p m}$ may be estimated as 


$$
\rho_{p m}=\frac{P}{c^{2}} \approx 10^{15} \mathrm{~g} / \mathrm{cm}^{3} .
$$

Atoms, nuclei and sub-nuclear particles do not have clear boundaries. What is the calculated value of proton radius? Tell the truth, the proton radius remains a puzzle today. However, there are indeed ambiguous physical scales. At present, it is generally accepted that the charge radius of protons is about

$(0.84 \sim 0.87) \times 10^{-13} \mathrm{~cm}$. According to [20], an equivalent radius $\tilde{R}_{p}$ of protons would be estimated as:

$$
\tilde{R}_{p} \sim \sqrt[3]{3 m_{p} / 4 \pi \rho_{m}} \approx 0.736 \times 10^{-13} \mathrm{~cm}
$$

which is obviously smaller than its charge radius and much larger than the generally accepted proton Compton wavelength. Therefore, the proton mass density $\rho_{p m}$ calculated from the normal proton's Compton scale size should be larger than the data obtained from the present measured value [20].

The smallest possible scale $r$ of a microscopic object being bound up with molecule, atom or nucleus must be related to the Heisenberg uncertainty principle:

$$
\Delta r \cdot \Delta p \geq \hbar,
$$

where $p(=m v)$ is its momentum. In history, both Davisson-Germer experiment and Heisenberg uncertainty principle were all published in 1927 [37] [38]. The Davisson-Germer experiment equivalently gave another expression of the uncertainty principle for microscopic objects:

$$
d \sin \theta_{n}=n \lambda>\lambda=h / p
$$

where $d$, for nickel, is line spacing, $\lambda$ is the wavelength associated with electrons with average momentum $p, n$ is an integer. A first-order maximum of intensity is to be expected at an angle $\theta_{1}(n=1)$ [37]. Written in the following form commonly used in discussing micro-objects:

$$
r \cdot p \geq \hbar
$$

Take notice

$$
r \geq \frac{\hbar}{m_{i} v}>\frac{\hbar}{m_{i} c}
$$

where $\hbar / m_{i} c$ is the Compton wavelength of the discussing micro-object $m_{i}$, its mean density $\rho_{i m}$ has the following relation

$$
\rho_{i m}=\frac{m_{i}}{\frac{4}{3} \pi r^{3}}<\frac{m_{i}}{\frac{4}{3} \pi\left(\frac{\hbar}{m_{i} c}\right)^{3}}\left(\sim \frac{3 m_{i}^{4} c^{3}}{4 \pi \hbar^{3}}\right) .
$$

An estimative figure might be carried out only for current matter-normal elementary particles, for instance, the "absolutely stable" proton $\left(m_{p}\right)$, or slowly decaying neutron $\left(m_{n}\right)$, the calculating results are the same in the order of magnitude:

$$
\rho_{i m}<\frac{3 m_{i}^{4} c^{3}}{4 \pi \hbar^{3}}\left(\sim 4.3 \times 10^{16} \mathrm{~g} / \mathrm{cm}^{3}\right),
$$


where $m_{i}$ is the mass of proton $\left(m_{p}\right)$ or the mass of neutron $m_{n}$. If substituting the mass of Higgs particle into Equation (14), the resulting upper limit would be much larger than Equation (14). Nevertheless, even if there would exist Higgs condensation (?) Which would be in vacuum, unlike the normal substances in our directly observable nature world.

Is there any inkling in nature to support Equation (14)? In recent decades in order to create and study vacuum excitations, vacuum phase transitions as well as a sheer surmise of mini black-hole, the ultra-relativistic heavy ions collision is considered as an effective way. The earlier data have been confirmed that QGP exist at an approximate extremely high temperature of 4 trillion $\left(2 \times 10^{12}\right)$ degree Celsius [39], the evaluating mass density of QGP fireball has almost reached $1 \mathrm{GeV} /\left(\mathrm{c}^{2} \cdot \mathrm{fm}^{3}\right)$, i.e. around $1.8 \times 10^{15} \mathrm{~g} / \mathrm{fm}^{3}$, which is less than the mass density of nucleon. Therefore QGP (quark soup) likes a big nucleus, and a nucleon likes a small bowl of quark soup. Consequently, the observational mass density of QGP in [39] agrees with Equation (11). This point would seem that "quark confinement" state and Equation (11) suggest following constraint:

Constraint 2-The average mass density of nucleon may be an unsurpassed upper limit for the normal matter in nature world.

Let Equation (11) / $\mathrm{M}^{2}<$ Equation (14), it turns that

$$
\frac{3 c^{6}}{32 \pi G^{3} M^{2}}<\frac{3 m_{i}^{4} c^{3}}{4 \pi \hbar^{3}},
$$

it is clear that

$$
M>m_{i} \cdot\left(\frac{\hbar c}{2 G m_{i}^{2}}\right)^{3 / 2} \sim 0.65 \times M_{\odot} .
$$

Seemingly, Constraint 2 or Equation (14) still could not stop the formation of black hole, however, a very important effect of combining two constraints reveals that there would exist an unattainability lower limit of total mass $0.65 \times M$. for a compact object made up of normal matter. If the "strong interaction gravitational constant" $G_{S}$ might be determined, the limit of mass of micro QGP would be determined also. Will there be any evidence? Aford, M. et al. took note "the observed absence of fast-spinning young stars" and tried to solve it [40]. Since the rotation speed is related to the mass density, the limit of the rotation speed indicates that there is an upper limit of the mass density. Therefore, it is absolutely impossible for late-type evolving celestial bodies to collapse to the singularity of centre ( $R=0)$, in other words, the singularity of centre $(R=0)$ in Schwarzschild metric has been eliminated.

\section{More New Physics}

Except for the generation and annihilation of matter and antimatter, we have never seen many substances created or disappeared during a very short time interval. Our common sense approach in the past may negate the possibility of the continuous creation or continuous extinction of vast substance. However, SR 
and GR combine with Heisenberg's uncertainty principle reveal some new physics knowledge which we still do not know so far:

1) In the traditional understanding of gravitational collapse, the collapsing body only collapses. Although the system has reached the instability of general relativity, the uncontrolled gravitational collapse would not stop, even if the scale size of the collapsed body approaches Schwarzschild radius, the total mass $M$ would not decrease. Constraint 1 and Equation (10) give another dynamic model of gravitational collapse, that is, with the decrease of scale size, the total mass $M$ of gravitational collapsed body also decreases, so as to ensure that compact intensity of the collapsed body always conforms to the laws within Constraint 1 and Equation (10).

2) Constraint 1 pointed out that this total mass reduction would be possible, but it is not yet clear about the detailed physical mechanisms and processes. At present, this article is just a guess that the new place might be vacuum, but it still does not known how to use astronomical observations to confirm this guess.

3) Around 1970s, Penrose, R. and Hawking, S. proved that under certain conditions (the validity of general relativity, the positive of energy, the universality of matter, and causality), gravitational collapse and physical singularity may be the inevitable fate of massive objects [41]-[47]. However, adding the Lorentz invariance of time-like metric, those concepts such as photon balls, event horizons, capture surfaces, white holes, wormholes, Einstein-Rosen bridges and so on would be all gone with negating the black hole. In addition, from Equation (14) and Equation (15), it may see that the big bang model of the universe still exists, and the expansion of the universe and the creation of matter also exist, and there does not exist the big bang model with the singularity of centre $(R=0)$ in nature. Equations (14) and (15) favor Gamow's fireball big bang model [48], which was based on and developed the "hypothèse de Patome primitif" (hypothesis of the primeval atom) proposed by Lemaître in 1931.

4) Take advantage of derivatives $\mathrm{d} M / \mathrm{d} t, \mathrm{~d} \rho / \mathrm{d} t, \mathrm{~d} R / \mathrm{d} t$, Constraint 1 and Equation (11) also manifests some new relations between relative rate of matter creation (or extinction) with the relative variable rates of scale $R$ and mean density $\rho$ :

During the expanding motion $(\mathrm{d} M / \mathrm{d} t>0, \mathrm{~d} R / \mathrm{d} t>0, \mathrm{~d} \rho / \mathrm{d} t<0)$ :

$$
\frac{2}{M} \frac{\mathrm{d} M}{\mathrm{~d} t}<\frac{2}{R} \frac{\mathrm{d} R}{\mathrm{~d} t}<-\frac{1}{\rho} \frac{\mathrm{d} \rho}{\mathrm{d} t},
$$

and during the collapsing motion $(\mathrm{d} M / \mathrm{d} t<0, \mathrm{~d} R / \mathrm{d} t<0, \mathrm{~d} \rho / \mathrm{d} t>0)$ :

$$
-\frac{2}{M} \frac{\mathrm{d} M}{\mathrm{~d} t}>-\frac{2}{R} \frac{\mathrm{d} R}{\mathrm{~d} t}>\frac{1}{\rho} \frac{\mathrm{d} \rho}{\mathrm{d} t} .
$$

5) It deserve to be mentioned that thinking of the expansion process of the universe, by means of Hubble's empirical law, link the velocity of recession of a distant celestial body and its distance from us $\mathrm{d} r / \mathrm{d} t=H_{0} r$ (where $H_{0}$ is the Hubble constant at present), it turns 


$$
\frac{\mathrm{d} \rho}{\mathrm{d} t}=\frac{\mathrm{d}(M / V)}{\mathrm{d} t}=\frac{\rho}{M} \frac{\mathrm{d} M}{\mathrm{~d} t}-3 \rho H_{0}
$$

Combining Equation (16) and Equation (18), One may find that in cosmic expansion, the upper limit of the relative matter creation rate and the lower limit of the relative mass density decreasing rate would be controlled by Hubble constant at present $H_{0}$.

$$
-\frac{1}{\rho} \frac{\mathrm{d} \rho}{\mathrm{d} t}>2 H_{0} \text { and } \frac{1}{M} \frac{\mathrm{d} M}{\mathrm{~d} t}<H_{0} .
$$

6) From the arguments mentioned above, we can see that the characteristics of the creation of matter in the process of expansion of universe are different from the steady-state cosmological model [49] [50], but the common point is that new substances are constantly created from vacuum. If vacuum is a physical channel for observing the disappearance of matter in the universe, it should also be a physical channel for the creation of matter, which may be one of the physical reasons for the expansion of the universe and producing dark matter.

\section{Remarks}

Some remarks should give as follows:

1) We study systems that would be large or small systems, or closed systems or open systems, and the impact of the environment on the systems is often very important. It is now recognized that a complete nature world (Universe) contains an observable universe incorporated with an infinite vacuum. With the passage of time, we now know that although vacuum has Lorentz invariance, but it is actually quite complicated. If some matter disappears or creates without a trace as the collapses or expands takes place, how do we describe the laws of conservation in the universe (including conservation of mass-energy, of baryons, of leptons, of electric charge and etc.)?

2) From a universal point of view, the law of conservation of energy should now be expressed as follows: The total energy of the Universe is conserved. If one part of the Universe obtains some form of energy, the other part will inevitably lose the same energy. No violations of this principle were found. In short, the observable universe and vacuum constitute an infinite, circular, and mutually transformed natural world.

3) Exactly as we know that Lorentz invariance is the inevitable outcome of two original postulates of SR. Bietenholz gave an introductory review about the ongoing search over more than 12 years for the evidence of Lorentz invariance violation (LIV) in ultro-high energy cosmic rays [39]. The extreme Lorentz factors with the order of $\gamma=1 / \sqrt{1-\beta^{2}} \sim 10^{11}$, but no LIV has been observed so far. So there's no need to waste any more time on the search for LIV.

4) Constraint 1, Constraint 2 and the combination of them expose the existence of new physics, and put forward a lot of open questions also:

a) In the observable universe, if matter would be created from vacuum, why does vacuum only create matter not antimatter? 
b) What is the spatial structural feature of new creating matter? Does it show some analogy with the structure of filaments in the supernova remnant or the reticular behavior in the very large-scale of an evolving universe?

c) May the forming new matter be one of the candidates for dark matter and dark energy?

d) Are we fortunate enough to make some morphological observations before and after the supernova explosion to confirm that if there might also be material creation in an explosion in a local area of the universe?

\section{Conclusion}

Theory without practice is empty theory, and practice without theoretical guidance is blind practice. Therefore, we must sum up practical experience by theory. On the basis of historical experience, supported by SR and GR, inspired by the latest developments in particle physics and high energy physics, the mystery of the singularity of Schwarzschild and the singularity of centre over the past century would be able to eliminate by adding only two physical restrictions on the Schwarzschild metric. This work fully proves the ancient Chinese motto: "Truth is simple", like the Ockham's Razor quote, which is worth thinking about.

\section{Acknowledgements}

This work was supported by the National Science Foundation of China Grant No. U1532260.

\section{Conflicts of Interest}

The author declares no conflicts of interest regarding the publication of this paper.

\section{References}

[1] Hawking, S. arXiv:1401.5761v1 [hep-th]

[2] Hawking, S. and Hertog, T. arXiv:1707.07702v3[hep-th]

[3] Mersini-Houghton, L. and Peiffer, H. arXiv:1406.1525 or arXiv:409.1837

[4] Vachaspati, T., et al. (2007) Physics Review D, 76, 024005. https://doi.org/10.1103/PhysRevD.76.024005

[5] Lemaître, G. (1933) Annales de la Société scientifique de Bruxelles, A53, 51-85.

[6] Einstein, A. and Rosen, N. (1935) Physics Review, 48, 73. https://doi.org/10.1103/PhysRev.48.73

[7] Kruskal, M. (1960) Physics Review, 119, 1743. https://doi.org/10.1103/PhysRev.119.1743

[8] Finkelstein, D. (1958) Physics Review, 110, 965. https://doi.org/10.1103/PhysRev.110.965

[9] Mei, X.C. (2011) International Journal of Astronomy and Astrophysics, 1, 109. https://doi.org/10.4236/ijaa.2011.13016 https://www.Scirp.org/journal/ijaa

[10] Mei, X.C. (2014) International Journal of Astronomy and Astrophysics, 4, 656. https://www.scirp.org/journal/ijaa 
https://doi.org/10.4236/ijaa.2014.44060

[11] Mei, X.C. (2013) Journal of Modern Physics, 4, 974-982.

https://doi.org/10.4236/jmp.2013.47131

https://www.scirp.org/journal/jmp

[12] Abbott, P., et al. (2016) Physical Review Letters, 116, Article ID: 061102. https://doi.org/10.1103/PhysRevLett.116.061102

[13] Klimenko, S., Vedovato, G., Drago, M., Salemi, F., Tiwari, V., Prodi, G.A., et al. (2016) Physical Review, D93, Article ID: 042004. https://doi.org/10.1103/PhysRevD.93.042004

[14] Pretorius, F. (2005) Physical Review Letters, 95, 121101. https://doi.org/10.1103/PhysRevLett.95.121101

[15] Campanelli, M., Lousto, C.O., Marronetti, P. and Zlochower, Y. (2006) Physical Review Letters, 96, Article ID: 111101. https://doi.org/10.1103/PhysRevLett.96.111101

[16] Baker, J., et al. (2006) Physical Review Letters, 96, Article ID: 111102. https://doi.org/10.1103/PhysRevLett.96.111102

[17] Hoyle, F. and Fowler, W. (1963) Nature, 197, 533. https://doi.org/10.1038/197533a0

[18] Avrett, E. (1976) Frontiers of Astrophysics. Harvard University Press, Cambridge, and London, 156.

[19] The STAR Collaboration (2017) Nature, 548, 62. https://doi.org/10.1038/nature23004

[20] Burkert1, V., et al. (2018) Nature, 557, 396.

[21] Weinberg, S. (1972) Gravitation and Cosmology: Principles and Applications of the General Theory of Relativity. John Wiley and Sons, New York, 347.

[22] Harms, R., et al. (1994) The Astrophysical Journal Letters, 435, L35. https://doi.org/10.1086/187588

[23] Wirsing, B. (2006) Discovery of Gamma Rays from the Edge of a Black Hole. Max Planck Society.

[24] Gaensler, B. (2011) Extreme Cosmos. New South Publishing, Sydney, 174.

[25] Shen, Z.Q., Lo, K.Y. and Liang, M.C. (2005) Nature, 438, 62. https://doi.org/10.1038/nature04205

[26] Burbidge, G. and Burbidge, E. (1969) Nature, 224, 21. https://doi.org/10.1038/224021a0

[27] Peach, J. (1969) Nature, 223, 1141. https://doi.org/10.1038/2231140a0

[28] Gaensler, B. (2011) Extreme Cosmos. New South Publishing, Sydney, 152.

[29] Osmer, P., et al. (1994) The Astrophysical Journal, 436, 678. https://doi.org/10.1086/174942

[30] Kaspi, S., et al. (2007) The Astrophysical Journal, 659, 997. https://doi.org/10.1086/512094

[31] Minkowski, H. (1952) The Principle of Relativity, a Collection of Original Memories. Dover Publications, New York, 73.

[32] Einstein, A. (1989) The Collected Papers of Albert Einstein. Vol. 2, Princeton University Press, Princeton, Doc 23.

[33] Einstein, A. (1955) The Meaning of Relativity. Princeton University Press, Princeton, 64 .

[34] Weinberg, S. (1972) Gravitation and Cosmology: Principles and Applications of the 
General Theory of Relativity. John Wiley and Sons, New York, 331.

[35] Weinberg, S. (1972) Gravitation and Cosmology: Principles and Applications of the General Theory of Relativity. John Wiley and Sons, New York, 332.

[36] Schmidt, C. and Sharma, S. (2017) Journal of Physics G: Nuclear and Particle Physics, 44, Article ID: 104002. https://doi.org/10.1088/1361-6471/aa824a

[37] Davisson, C. and Germer, L. (1927) Nature, 119, 558. https://doi.org/10.1038/119558a0

[38] Heisenberg, W. (1927) Z. Physik, 43, 172. https://doi.org/10.1007/BF01397280

[39] Bietenholz, W. (2011) Physics Report, 505, 145. https://doi.org/10.1016/j.physrep.2011.04.002

[40] Aford, M., et al. (2012) Physical Review D, 85, Article ID: 044051. https://doi.org/10.1103/PhysRevD.85.044051

[41] Penrose, R. (1965) Physical Review Letters, 14, 57. https://doi.org/10.1103/PhysRevLett.14.57

[42] Hawking, S. (1966) Proceedings of the Royal Society, 294A, 511.

[43] Hawking, S. (1966) Proceedings of the Royal Society, 295A, 490.

[44] Hawking, S. (1967) Proceedings of the Royal Society, 300A, 187.

[45] Hawking, S. (1967) Proceedings of the Royal Society, 308A, 433.

[46] Hawking, S. and Penrose, R. (1970) Proceedings of the Royal Society, A314, 529. https://doi.org/10.1098/rspa.1970.0021

[47] Penrose, R. (1969) La Rivista del Nuovo Cimento, 1, 252.

[48] Gamow, G. (1946) Physical Review, 70, 572. https://doi.org/10.1103/PhysRev.70.572.2

[49] Hoyle, F. (1948) Monthly Notices of the Royal Astronomical Society, 108, 372. https://doi.org/10.1093/mnras/108.5.372

[50] Hoyle, F. (1949) Monthly Notices of the Royal Astronomical Society, 109, 365. https://doi.org/10.1093/mnras/109.3.365 